\title{
An auxiliary-field quantum Monte Carlo study of the chromium dimer
}

Wirawan Purwanto

College of William and Mary

Shiwei Zhang

College of William and Mary

Henry Krakauer

College of William and Mary

Follow this and additional works at: https://scholarworks.wm.edu/aspubs

\section{Recommended Citation}

Purwanto, W., Zhang, S., \& Krakauer, H. (2015). An auxiliary-field quantum Monte Carlo study of the chromium dimer. The Journal of chemical physics, 142(6), 064302.

This Article is brought to you for free and open access by the Arts and Sciences at W\&M ScholarWorks. It has been accepted for inclusion in Arts \& Sciences Articles by an authorized administrator of W\&M ScholarWorks. For more information, please contact scholarworks@wm.edu. 


\title{
An auxiliary-field quantum Monte Carlo study of the chromium dimer
}

\author{
Wirawan Purwanto, ${ }^{\text {a) }}$ Shiwei Zhang, and Henry Krakauer \\ Department of Physics, College of William and Mary, Williamsburg, Virginia 23187-8795, USA
}

(Received 13 October 2014; accepted 15 January 2015; published online 9 February 2015)

\begin{abstract}
The chromium dimer $\left(\mathrm{Cr}_{2}\right)$ presents an outstanding challenge for many-body electronic structure methods. Its complicated nature of binding, with a formal sextuple bond and an unusual potential energy curve (PEC), is emblematic of the competing tendencies and delicate balance found in many strongly correlated materials. We present an accurate calculation of the PEC and ground state properties of $\mathrm{Cr}_{2}$, using the auxiliary-field quantum Monte Carlo (AFQMC) method. Unconstrained, exact AFQMC calculations are first carried out for a medium-sized but realistic basis set. Elimination of the remaining finite-basis errors and extrapolation to the complete basis set limit are then achieved with a combination of phaseless and exact AFQMC calculations. Final results for the PEC and spectroscopic constants are in excellent agreement with experiment. (C) 2015 AIP Publishing LLC. [http://dx.doi.org/10.1063/1.4906829]
\end{abstract}

The chromium dimer $\left(\mathrm{Cr}_{2}\right)$ is a strongly correlated molecule which poses a formidable challenge to even the most accurate many-body methods. It features a formal sextuple bond, with a weak binding energy $(\sim 1.5 \mathrm{eV})$, a short equilibrium bond length $(\sim 1.7 \AA)$, and an unusual "shoulder" structure in its potential energy curve (PEC). ${ }^{1-3}$ The ground state of $\mathrm{Cr}_{2}$ is highly multiconfigurational, and proper theoretical description requires an accurate treatment of the strong $3 d$ electron correlations (both static and dynamic). The nature of the $\mathrm{PEC}$ in $\mathrm{Cr}_{2}$ is representative of the competing tendencies separated by small energy differences seen in many strongly correlated materials. Because of the fundamental and technological significance of such materials, improving our abilities for accurate calculations in strongly correlated systems is one of the most pressing needs in condensed matter physics and quantum chemistry.

Standard quantum chemistry methods, such as density functional theory (DFT), Hartree-Fock (HF), and post-HF methods such as single-reference second-order Møller-Plesset perturbation theory (MP2) and single-reference coupled cluster with singles, doubles, and perturbative triples $[\operatorname{CCSD}(\mathrm{T})]$, all fail to describe the correct binding of $\mathrm{Cr}_{2}$. Representative standard quantum chemistry results are shown in Fig. 1. As often is the case, the DFT results vary greatly, depending on the choice of exchange-correlation functional. There have also been numerous attempts to calculate the PEC of $\mathrm{Cr}_{2}$ using sophisticated multireference quantum chemistry methods, ${ }^{4-9}$ including the complete active space second-order perturbation theory (CASPT2) ${ }^{10-12}$ and, more recently, CASPT2 based on a large density matrix renormalization group (DMRG) reference wave function (DMRG-CASPT2)..$^{13}$ These calculations obtain qualitatively correct binding, but the results are sensitive to choice of active space and/or basis set. Standard quantum Monte Carlo (QMC) approaches ${ }^{14,15}$ have also been severely challenged. A recent fixed-node diffusion Monte Carlo (DMC) study, which examined the use of a variety of single- and

\footnotetext{
a)Electronic address: wirawan0@gmail.com
}

multi-determinant trial wave functions, did not obtain satisfactory binding (indeed the molecular energy was found to be higher than the sum of two isolated atoms). ${ }^{16}$ All these underline the extreme challenge in achieving an accurate theoretical description of the $\mathrm{Cr}_{2}$ PEC.

In this paper, we present calculations of the $\mathrm{Cr}_{2}$ PEC and ground-state properties using the auxiliary-field quantum Monte Carlo (AFQMC) method. ${ }^{17-20}$ We first describe exact free-projection (FP) AFQMC calculations, where we release the usual phaseless approximation ${ }^{17}$ used to control the phase/sign problem. ${ }^{20}$ The results are then extrapolated to the complete basis set (CBS) limit using a combination of phaseless and exact AFQMC calculations. Final results for the PEC and spectroscopic constants are in excellent agreement with experiment.

AFQMC obtains ground-state properties by stochastically sampling the many-body ground-state wave function in the space of Slater determinants, expressed in a chosen oneparticle basis. ${ }^{17-20}$ It has modest polynomial scaling with system size $N\left[O\left(N^{3}\right)\right.$ or $\left.O\left(N^{4}\right)\right]$ rather than the exponential scaling of CI calculations, or the high-order polynomial scaling of typical quantum chemistry many-body methods. The FPAFQMC, , $^{21,22}$ which leaves the fermion sign/phase problem uncontrolled,$^{20}$ is exact but has exponential scaling due to rapidly increasing stochastic noise with projection imaginarytime. The AFQMC phaseless approximation (ph-AFQMC) ${ }^{17}$ was introduced to control this, resulting in a practical method which restores the low computational scaling. The method has been demonstrated to yield accurate results in many atomic, molecular, cluster, and extended systems. ${ }^{17-19,23,24} \mathrm{For}_{2}$, we have found that the current implementation of the phaseless approximation (using standard single- or multi-determinant trial wave functions), while leading to a qualitatively correct PEC, exhibits noticeable systematic error in the binding energy. To eliminate the residual systematic errors, we are able to carry out exact, large-scale FP-AFQMC calculations using a moderate-sized but realistic basis set. These exact results are used to benchmark other many-body methods, including 


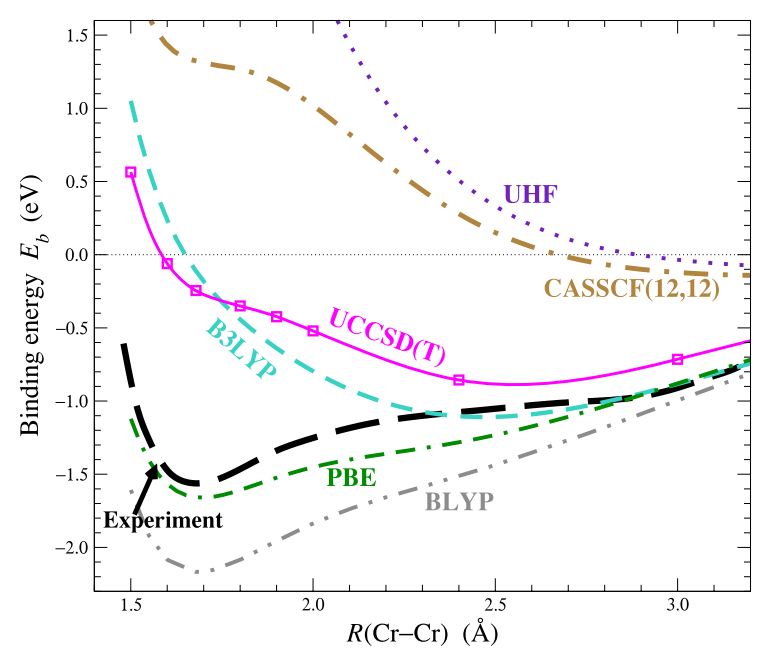

FIG. 1. Representative results of the $\mathrm{Cr}_{2}$ PEC from standard quantum chemistry calculations. Shown are results from UHF, CASSCF with 12 active electrons and 12 active orbitals, $\operatorname{CCSD}(\mathrm{T})$ with UHF reference wave function [UCCSD(T)], and DFT with various exchange-correlation functionals (B3LYP, ${ }^{37}$ PBE, ${ }^{38}$ BLYP $^{39,40}$ ). Most calculations use the cc-pwCVTZ-DK basis set, except UCCSD(T), where extrapolation to the CBS limit was done, as described later in the text. Experimental PEC was taken from Ref. 3.

ph-AFQMC as well as previously published results. In a final step, the results are combined with ph-AFQMC calculations with large basis sets to obtain an accurate PEC in the CBS limit.

The AFQMC calculations reported here employ standard quantum chemistry Gaussian type orbital basis sets. ${ }^{25}$ Our calculations employed the Douglas-Kroll-Hess scalarrelativistic all-electron Hamiltonian, with core-valence correlation-consistent Gaussian basis sets, cc-pwCV $x Z-D K$, with $x=3,4,5$. (We will hereafter refer to these as TZ, QZ, and 5Z, respectively.) For a chosen basis, AFQMC thus treats the same Hamiltonian as that of a corresponding many-body quantum chemistry calculation, allowing, for example, direct comparisons of absolute total energies. This was done below with DMRG calculations, where results using a small splitvalence (SV) basis ${ }^{26}$ were available. ${ }^{27,28} \mathrm{AFQMC}$ projects the ground state starting from a trial wave function $\Psi_{\mathrm{T}}$, which is also used in the mixed-estimator to compute the groundstate energy and additionally in ph-AFQMC to control the fermionic sign/phase problem. We used two choices of $\Psi_{\mathrm{T}}$, the unrestricted Hartree-Fock (UHF) and truncated complete active space self-consistent field (t-CASSCF) as in our earlier work. ${ }^{23} \operatorname{CASSCF}(12,12)$ was used, which fully correlates 12 active electrons in 12 orbitals derived from the $3 d$ and $4 s$ atomic states. The CASSCF wave function is truncated such that the weight (squared coefficient) of the retained determinants is $\sim 90 \%-92 \%$ of the total. This particular way of choosing the t-CASSCF $\Psi_{\mathrm{T}}$ becomes increasingly expensive as the atoms are stretched from the equilibrium bond length; for larger bond lengths, broken spin symmetry $\mathrm{UHF} \Psi_{\mathrm{T}}$ was used for FP-AFQMC, as discussed below. All calculations used the frozen-core approximation, ${ }^{29}$ freezing neon-core orbitals calculated at a lower level of theory (HF here). The frozencore Hamiltonian one- and two-body matrix elements and $\Psi_{\mathrm{T}}$ were obtained using outputs from modified quantum chemistry codes, NWCHEM ${ }^{30}$ and GAMESS. ${ }^{31}$

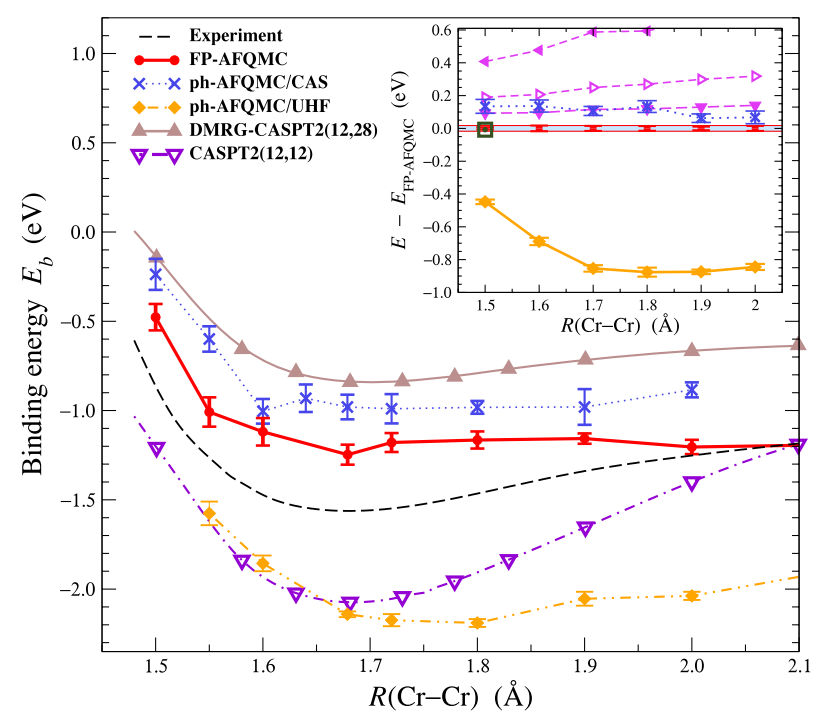

FIG. 2. Benchmarking the $\mathrm{Cr}_{2}$ PECs in finite basis sets, near experimental equilibrium. The main figure shows the calculated binding energy with the cc-pwCVTZ-DK basis, comparing exact FP-AFQMC PEC to ph-AFQMC, as well as DMRG-CASPT2 and CASPT2 (see text) from Ref. 13. The experimental PEC is also shown for reference. The inset compares total energies (with respect to exact FP-AFQMC) for DMRG and ph-AFQMC for the small SV basis. ${ }^{26}$ AFQMC results have the same symbols as in the main figure. The $M \rightarrow \infty$ extrapolated DMRG result ${ }^{28}$ is shown for $R=1.5 \AA$ as an empty square. Finite- $M$ DMRG results ${ }^{27}$ for various geometries are also shown: $M=1600$ (down triangle, filled), $M=800$ (right triangle, empty), and $M=400$ (left triangle, filled).

Figure 2 presents benchmark results of the calculated $\mathrm{Cr}_{2}$ PECs near experimental equilibrium $(R \lesssim 2.1 \AA)$, where results are available for all computational methods. The main figure compares AFQMC to CASPT2 calculations using the realistic TZ basis set. The inset compares AFQMC to DMRG calculations for the small SV basis. The binding energy is given by $E_{b} \equiv E_{\mathrm{mol}}-2 E_{\text {atom }}$, where the molecular and atomic energies are calculated with the same method. The FP-AFQMC calculations are exact for the chosen basis. (While we have included the experimental PEC in the figure for reference, it should not be directly compared to FP-AFQMC using the TZ basis. Extrapolation to the CBS limit is discussed below.) These calculations were done with $\sim 10^{5}$ or more walkers, with an imaginary-time projection of 400-500 steps and a time-step of $0.02 E_{\mathrm{h}}^{-1}$, requiring significant computing resources. Energy measurement was carried out after the total energy has converged as a function of the projection time; alternative analysis across different subsections of the walker population was also performed to monitor the consistency of the statistical error bar. We have verified that the Trotter error in the calculated FP-AFQMC binding energy is smaller than the statistical error bar. Phaseless AFQMC results are also shown, using UHF and t-CASSCF $\Psi_{\mathrm{T}}$, denoted ph-AFQMC/UHF and ph-AFQMC/CAS, respectively. These were done with $\sim 2000$ walkers and are essentially in the zero Trotter time-step limit. The ph-AFQMC/CAS PEC shows a small non-parallelity error (NPE) and is $\sim 0.2 \mathrm{eV}$ above the exact result. The ph-AFQMC/UHF PEC shows larger discrepancies, lying below FP-AFQMC by $\sim 0.6-1.0 \mathrm{eV}$. (Since ph$\mathrm{AFQMC}$ is non-variational, ${ }^{32}$ this is possible, especially with a poor $\Psi_{\mathrm{T}}$.) These AFQMC results are compared to two 
perturbative CASPT2 calculations (both results were obtained from Fig. 2 of Ref. 13). The PEC labeled CASPT2(12,12) is based on a $\operatorname{CASSCF}(12,12)$ reference wave function. The CASPT2 $(12,12)$ PEC is overbound by $\sim 1 \mathrm{eV}$, indicating the inadequacy of $\operatorname{CASSCF}(12,12)$ as the zeroth-order wave function. The DMRG-CASPT2 $(12,28)$ PEC was obtained with the DMRG self-consistent field reference wave function for an active space of 12 electrons and 28 orbitals, much larger than that of the $\operatorname{CASSCF}(12,12)$. The DMRG procedure for the full TZ PEC, however, kept only $M=512$ states. (In Ref. 13, DMRG-CASPT2 results converged with respect to $M$ were presented at equilibrium for $5 \mathrm{Z}$ basis.) Converging the DMRG to $M \rightarrow \infty$ would lower the DMRG-CASPT2 PEC and could make it closer to the FP-AFQMC result.

The inset in Fig. 2 compares the total energy from FPAFQMC, ph-AFQMC, and DMRG calculations. (The SV basis benchmark calculations were done with a non-relativistic Hamiltonian with 12 core electrons frozen. With this small basis, the molecule is not bound.) Similar to the TZ-basis results in the main figure, ph-AFQMC/CAS lies above the exact FP-AFQMC curve by $\lesssim 0.15 \mathrm{eV}$ with a small NPE $\lesssim 0.08 \mathrm{eV}$, while ph-AFQMC/UHF shows larger errors. At $R=1.5 \AA$, the $(M \rightarrow \infty)$ DMRG result ${ }^{28}$ agrees with exact FP-AFQMC to within the stochastic error of the latter: $E_{\mathrm{DMRG}}-E_{\mathrm{FP}-\mathrm{AFQMC}}$ $=-8 \pm 7 \mathrm{meV}$. For other $R$, only finite- $M$ DMRG results are available, ${ }^{27}$ and these are also shown for comparison; they provide improving variational bounds of the total energy as $M$ increases.

The benchmark results in Fig. 2 illustrate the importance of $\Psi_{\mathrm{T}}$ for ph-AFQMC in the strongly correlated $\mathrm{Cr}_{2}$. Although the UHF and t-CASSCF wave functions have similar variational energies, t-CASSCF is a better $\Psi_{\mathrm{T}}$ because it more accurately describes the multiconfigurational nature of the ground state and, unlike UHF, does not break spin symmetry (to within small truncation error). For example, at stretched geometries $R \gtrsim 1.9 \AA$, UHF has a lower variational energy than the t-CASSCF $\Psi_{\mathrm{T}}$ at $90 \%$ total weight; but the UHF $\Psi_{\mathrm{T}}$ has large spin-contamination $S^{2} \gtrsim 5$. This leads to significant errors in ph-AFQMC/UHF and long imaginary-time equilibration. ${ }^{33}$ The AFQMC/CAS approach, on the other hand, becomes increasingly expensive as the atoms are stretched from the equilibrium geometry, because the number of the required determinants in $\Psi_{\mathrm{T}}$ grows rapidly. (At $2.0 \AA$, for example, a $92 \%$ cut retains $\sim 1800$ determinants.) For FPAFQMC, which is not biased by $\Psi_{\mathrm{T}}$, calculations at larger bond lengths were carried out by initializing an approximately spin-pure walker population using an aggressively truncated t-CASSCF wave function, while the energy mixed-estimator was evaluated using the UHF $\Psi_{\mathrm{T}}$. This approach reduces the time to equilibrate FP-AFQMC.

The final CBS correction, which is to be added to the cc-pwCVTZ-DK FP-AFQMC results in Fig. 2, consists of a (small) HF contribution plus a correlation contribution $\Delta E_{b}{ }^{34}$ The latter is given by the shaded curve in Fig. 3. It was obtained by extrapolating TZ and QZ results, with cross-check from $5 Z$ calculations, as follows. For $R<2.0 \AA$, we performed phAFQMC calculations using both UHF and t-CASSCF trial wave functions to extrapolate to the CBS limit. ${ }^{34,35}$ Although the ph-AFQMC/UHF PEC lies below the exact result, while

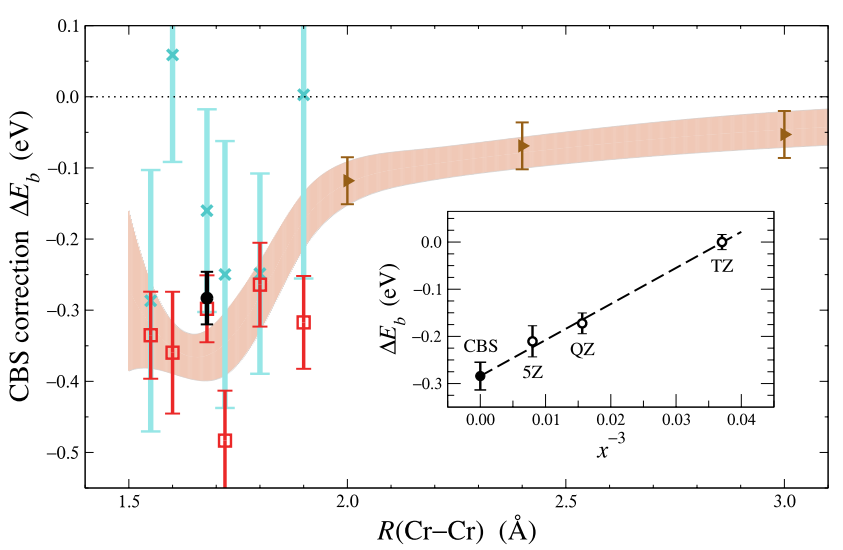

FIG. 3. CBS correction to the correlation contribution of the binding energy, with respect to cc-pwCV $x \mathrm{Z}-\mathrm{DK}$, as a function of $\mathrm{Cr}-\mathrm{Cr}$ distance. The red open squares are from ph-AFQMC/UHF, blue crosses from ph-AFQMC/CAS, while the brown triangles are from free projection. The ph-AFQMC/UHF and free projection results were used to fit a smooth CBS correction curve, as described in the text. The final CBS correction is shown by the shaded band in the main figure, where the shading width represents combined stochastic and fitting errors. The inset verifies the accuracy of the TZ-QZ extrapolation, using a ph-AFQMC/UHF $5 Z$ calculation at $1.68 \AA$ (the black filled circle in the main figure), where good linearity is seen in the plot of cc-pwCVxZ-DK results vs. the inverse cube of the basis set cardinal number $x$.

ph-AFQMC/CAS lies above, their respective $\Delta E_{b}$ are in reasonably good agreement. The UHF results, which have considerably smaller statistical error bars, are used to obtain the smooth fit. We also performed a ph-AFQMC/UHF 5Z calculation at $R=1.68 \AA$ to check the accuracy of the TZ-QZ extrapolation, as shown in the inset. For $R \geq 2.0 \AA$, we used an alternative approach to obtain $\Delta E_{b}$, since both ph-AFQMC/UHF and ph-AFQMC/CAS have some difficulties in this region, as discussed earlier. FP-AFQMC/UHF TZ and QZ calculations were performed for a larger frozen core, which also freezes the semicore $3 s$ and $3 p$ orbitals. For larger bond lengths, the absence of semicore correlation effects has negligible effect on $\Delta E_{b}$, as we confirmed with UCCSD(T) [CCSD(T) with UHF reference wave function] TZ and QZ calculations. With fewer correlated electrons, stochastic errors were reduced, allowing us to extract $\Delta E_{b}$ with FP-AFQMC. (We also found that $\Delta E_{b}$ converged well before full equilibration with both the $\mathrm{TZ}$ and QZ bases.) We used a quadratic ansatz to fit $\Delta E_{b}$ for $R<2.0 \AA$, a $(1 / R)$ function for $R \geq 2.0 \AA$, and a spline joining the two regions to yield the final $\Delta E_{b}$ for the entire PEC.

The CBS-extrapolated FP-AFQMC PEC, shown in Fig. 4, is generally in excellent agreement with experiment, except for the shoulder region, which is discussed further below. We also applied the same CBS extrapolation correction to the ph-AFQMC/CAS PEC from Fig. 2, shown as "ph-AFQMC" PEC in Fig. 4. The corresponding spectroscopic constants, obtained from both FP- and ph-AFQMC, are compared to experiment in Table I. The UHF, CASSCF $(12,12)$, and UCCSD(T) PECs (reproduced from Fig. 1) do not show binding [although $\operatorname{UCCSD}(\mathrm{T})$ has an outer well near $2.7 \AA$. . Both $\operatorname{CASSCF}(12,12)$ and $\mathrm{UCCSD}(\mathrm{T})$ evidence a plateau-like feature at short $\mathrm{Cr}-\mathrm{Cr}$ distance, however. As previously seen in other applications, the ph-AFQMC recovers from a qualitatively incorrect $\Psi_{\mathrm{T}}$ from UHF or CASSCF $(12,12)$. Although the ph-AFQMC/CAS is somewhat underbound, the equilibrium bond length and 


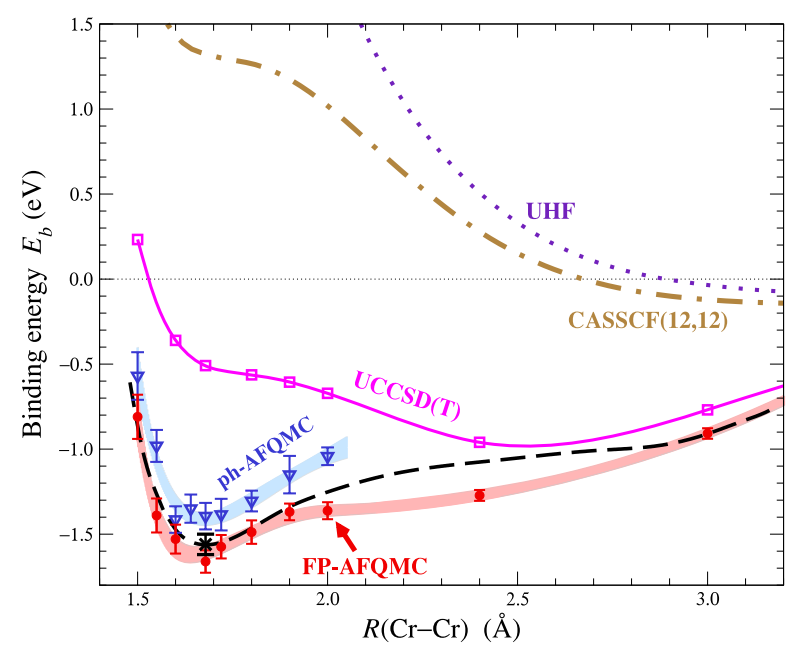

FIG. 4. CBS extrapolated ph-AFQMC and exact FP-AFQMC PECs compared to experiment (dashed black line). Results from several standard quantum chemistry methods are also shown for reference. The experimental binding energy ${ }^{2}$ at the experimental bond distance ${ }^{1}$ is shown as a black " $\times$ " symbol together with the error bar reflecting its uncertainty.

vibrational frequency agree very well with experiment as shown in Table I. The FP-AFQMC result is seen to lie somewhat lower than experiment in the shoulder region $\simeq 2.0-2.7 \AA$, which is where the experimental $\mathrm{PEC}^{3}$ has the greatest uncertainty. The experimental PEC was based on high-resolution photoelectron spectra of $\mathrm{Cr}_{2}^{-}$, which showed 29 vibrationally resolved transitions to the neutral $\mathrm{Cr}_{2}$ ground state. As noted in Ref. 3, there were large gaps in the vibrational data between 3040 and $4880 \mathrm{~cm}^{-1}$, which insufficiently constrained the shape of the potential in this region. The possibility was stated that the true PEC could actually have a shallow minimum where the experimentally fitted PEC exhibits a shoulder. Future theoretical study, with reduced stochastic uncertainty and at more bondlengths, is warranted to further determine the shape of the PEC in this region.

In summary, we have presented an accurate calculation of the PEC and spectroscopic properties of $\mathrm{Cr}_{2}$, using the AFQMC method. Unconstrained, exact AFQMC calculations were first carried out for a medium-sized but realistic basis set. Elimination of the remaining finite-basis errors and extrapolation to the CBS limit were then achieved with a combination of phaseless and FP AFQMC calculations. This hybrid approach

TABLE I. Spectroscopic constants of $\mathrm{Cr}_{2}$ computed using phaseless and free-projection AFQMC methods, extrapolated to the CBS limit. $E_{b}$ is the molecular binding energy (zero-point energy has been removed from the experimental value); $R_{0}$ is the equilibrium bond length; and $\omega_{e}$ is the harmonic vibrational frequency.

\begin{tabular}{lccc}
\hline \hline Method & $E_{b}(\mathrm{eV})$ & $R_{0}(\AA)$ & $\omega_{e}\left(\mathrm{~cm}^{-1}\right)$ \\
\hline ph-AFQMC & $-1.42(4)$ & $1.68(2)$ & $520(59)$ \\
FP-AFQMC & $-1.63(5)$ & $1.65(2)$ & $552(93)$ \\
\hline Experiment & $-1.56(6)^{\mathrm{a}}$ & $1.6788^{\mathrm{b}}$ & $480.6(5)^{\mathrm{c}}$ \\
& $-1.47(5)^{\mathrm{d}}$ & & \\
\hline \hline
\end{tabular}

${ }^{\mathrm{a}}$ Reference 2 .

${ }^{\mathrm{b}}$ Reference 1 .

${ }^{\mathrm{c}}$ Reference 3 .

${ }^{\mathrm{d}}$ Reference 36 . enabled us to obtain one of the most accurate theoretical results of $\mathrm{Cr}_{2}$ ground-state properties obtained to date, which is in excellent agreement with experiment.

This work was supported by DOE (No. DE-FG0209ER16046), NSF (No. DMR-1409510), and ONR (No. N000141211042). We acknowledge a DOE CMCSN grant (No. DE-FG02-11ER16257) for facilitating stimulating interactions. An award of computer time was provided by the Innovative and Novel Computational Impact on Theory and Experiment (INCITE) program, using resources of the Oak Ridge Leadership Computing Facility (Titan) at the Oak Ridge National Laboratory, which is supported by the Office of Science of the U.S. Department of Energy under Contract No. DE-AC05-00OR22725. Some of the earlier computing was performed on Blue Waters, which is supported by the National Science Foundation (Award No. OCI 07-25070) and the state of Illinois. Blue Waters is a joint effort of the University of Illinois at Urbana-Champaign and its National Center for Supercomputing Applications. We also acknowledge the computing support from the Center for Piezoelectrics by Design. The authors would like to thank Yudistira Virgus, Eric J. Walter, and Simone Chiesa for many useful discussions.

${ }^{1}$ V. E. Bondybey and J. H. English, Chem. Phys. Lett. 94, 443 (1983).

${ }^{2}$ B. Simard, M.-A. Lebeault-Dorget, A. Marijnissen, and J. J. ter Meulen, J. Chem. Phys. 108, 9668 (1998).

${ }^{3}$ S. M. Casey and D. G. Leopold, J. Phys. Chem. 97, 816 (1993).

${ }^{4}$ H. Stoll and H.-J. Werner, Mol. Phys. 88, 793 (1996).

${ }^{5}$ H. Dachsel, R. J. Harrison, and D. A. Dixon, J. Phys. Chem. A 103, 152 (1999).

${ }^{6}$ C. Angeli, R. Cimiraglia, and J.-P. Malrieu, J. Chem. Phys. 117, 9138 (2002).

${ }^{7}$ C. Angeli, B. Bories, A. Cavallini, and R. Cimiraglia, J. Chem. Phys. 124, 054108 (2006).

${ }^{8}$ P. Celani, H. Stoll, H.-J. Werner, and P. J. Knowles, Mol. Phys. 102, 2369 (2004).

${ }^{9}$ T. Müller, J. Phys. Chem. A 113, 12729 (2009).

${ }^{10}$ K. Andersson, Chem. Phys. Lett. 237, 212 (1995).

${ }^{11}$ B. O. Roos and K. Andersson, Chem. Phys. Lett. 245, 215 (1995).

${ }^{12}$ B. O. Roos, Collect. Czech. Chem. Commun. 68, 265 (2003).

${ }^{13}$ Y. Kurashige and T. Yanai, J. Chem. Phys. 135, 094104 (2011).

${ }^{14}$ R. J. Needs, M. D. Towler, N. D. Drummond, and P. López Rios, J. Phys.: Condens. Matter 22, 023201 (2010), also see the references therein.

${ }^{15}$ J. Kolorenč and L. Mitas, Rep. Prog. Phys. 74, 026502 (2011), also see the references therein.

${ }^{16}$ K. Hongo and R. Maezono, Int. J. Quantum Chem. 112, 1243 (2012).

${ }^{17}$ S. Zhang and H. Krakauer, Phys. Rev. Lett. 90, 136401 (2003).

${ }^{18}$ W. A. Al-Saidi, S. Zhang, and H. Krakauer, J. Chem. Phys. 124, 224101 (2006).

${ }^{19}$ M. Suewattana, W. Purwanto, S. Zhang, H. Krakauer, and E. J. Walter, Phys. Rev. B 75, 245123 (2007).

${ }^{20} \mathrm{~S}$. Zhang, in Emergent Phenomena in Correlated Matter, edited by E. Pavarini, E. Koch, and U. Schollwöck (Forschungszentrum Jülich Zentralbibliothek, Verlag Jülich, 2013), Chap. 15, ISBN: 3893368841, URL: http: //juser.fz-juelich.de/record/137827.

${ }^{21}$ W. Purwanto, H. Krakauer, and S. Zhang, Phys. Rev. B 80, 214116 (2009).

${ }^{22}$ H. Shi and S. Zhang, Phys. Rev. B 88, 125132 (2013).

${ }^{23}$ W. Purwanto, S. Zhang, and H. Krakauer, J. Chem. Phys. 130, 094107 (2009).

${ }^{24}$ Y. Virgus, W. Purwanto, H. Krakauer, and S. Zhang, Phys. Rev. Lett. 113, 175502 (2014).

${ }^{25}$ K. L. Schuchardt, B. T. Didier, T. Elsethagen, L. Sun, V. Gurumoorthi, J. Chase, J. Li, and T. L. Windus, J. Chem. Inf. Model. 47, 1045 (2007).

${ }^{26}$ A. Schäfer, H. Horn, and R. Ahlrichs, J. Chem. Phys. 97, 2571 (1992).

${ }^{27}$ Y. Kurashige and T. Yanai, J. Chem. Phys. 130, 234114 (2009).

${ }^{28}$ S. Sharma and G. K.-L. Chan, J. Chem. Phys. 136, 124121 (2012).

${ }^{29}$ W. Purwanto, S. Zhang, and H. Krakauer, J. Chem. Theory Comput. 9, 4825 (2013). 
${ }^{30}$ M. Valiev, E. Bylaska, N. Govind, K. Kowalski, T. Straatsma, H. van Dam, D. Wang, J. Nieplocha, E. Apra, T. Windus et al., Comput. Phys. Commun. 181, 1477 (2010)

${ }^{31}$ M. W. Schmidt, K. K. Baldridge, J. A. Boatz, S. T. Elbert, M. S. Gordon, J. H. Jensen, S. Koseki, N. Matsunaga, K. A. Nguyen, S. J. Su et al., J. Comput. Chem. 14, 1347 (1993).

${ }^{32}$ J. Carlson, J. E. Gubernatis, G. Ortiz, and S. Zhang, Phys. Rev. B 59, 12788 (1999).

${ }^{33}$ W. Purwanto, W. A. Al-Saidi, H. Krakauer, and S. Zhang, J. Chem. Phys. 128, 114309 (2008).
${ }^{34}$ W. Purwanto, H. Krakauer, Y. Virgus, and S. Zhang, J. Chem. Phys. 135, 164105 (2011).

${ }^{35}$ T. Helgaker, W. Klopper, H. Koch, and J. Noga, J. Chem. Phys. 106, 9639 (1997).

${ }^{36}$ K. Hilpert and K. Ruthardt, Ber. Bunsenges. Physik. Chem. 91, 724 (1987).

${ }^{37}$ P. Stephens, F. J. Devlin, C. F. Chabalowski, and M. J. Frisch, J. Phys. Chem. 98, 11623 (1994).

${ }^{38}$ J. P. Perdew, K. Burke, and M. Ernzerhof, Phys. Rev. Lett. 77, 3865 (1996).

${ }^{39}$ A. D. Becke, Phys. Rev. A 38, 3098 (1988).

${ }^{40}$ C. Lee, W. Yang, and R. G. Parr, Phys. Rev. B 37, 785 (1988). 